\title{
Transient Free Convective Flow over a Vertical Cone Embedded in a Thermally Stratified Medium
}

\author{
Bapuji Pullepu and Y. Immanuel
}

\begin{abstract}
Unsteady natural convection flow of a viscous and incompressible flow over a vertical cone immersed in a stable thermally stratified medium is theoretically studied in this paper. The dimensionless coupled partial differential boundary layer equations are solved numerically using an efficient and unconditionally stable finite-difference scheme of Crank-Nicolson type. The effects of Prandtl number and stratification parameter on the velocity and temperature profiles on the flow and heat transfer characteristics have been determined and discussed in detail. The present results are compared with available results from the open literature and are found to be in good agreement. We notice that natural convection flow over a vertical surface embedded in porous media also occurs in several engineering problems such as those encountered in the design of pebble-bed nuclear reactors, catalytic reactors, and compact heat exchangers, in geothermal energy conversion, in the use of fibrous materials, in the thermal insulation of buildings, in the heat transfer from storage of agricultural products which generate heat as a result of metabolism, in petroleum reservoirs, in nuclear wastes, etc.
\end{abstract}

Keywords--- Finite Difference, Free Convection, Stratified Medium, Unsteady, Vertical Cone

\section{INTRODUCTION}

$\mathrm{N}$ ATURAL convection flows under influence of gravitational force have been investigated most extensively because they frequently occur in nature as well as in science and engineering applications. When a heated surface is in contact with the fluid, the result of temperature difference causes buoyancy force, which induces the natural convection. Many free convection processes occur in environment with temperature stratification. Good examples are closed containers and environmental chambers with heated walls. A room, which is heated by electrical wires embedded in the ceiling may also be thermally stratified. A room fire with an open door or window through which fresh air is supplied near the bottom offers another example of a thermally stratified situation. The atmosphere itself is thermally stratified, as is the ocean. Stratification of the fluid arises due to a temperature variation, concentration differences or the presence of different fluids. Thermal stratification is important in lakes, rivers, sea, condensers of power plants and various industrial units. This type of flow is also of fundamental importance due to its

Bapuji Pullepu, Assistant Professsor, SRM University, Chennai, India.Email:bapujip@yahoo.com

Y. Immanuel, AssistantProfesssor, Sathyabama University, Chennai, India.E-mail:y_immanuel@yahoo.com possible applications in heat exchanger devices, filtration and petroleum reservoirs.

Many investigations have been developed from the past four decades for the free convection flow from various geometries (plane or axi-symmetrical shape bodies) in thermally stratified medium. Cheesewright [1] discussed a theoretical investigation of free convection from a vertical flat plate in non-isothermal surroundings. He obtained similarity solutions of the governing equations dealing with various types of non-uniform ambient temperature distributions. Yang et al. [2] studied natural convection heat transfer from a nonisothermal vertical flat plate immersed in a thermal stratified medium and similarity solutions have been carried out for a wide range of wall and ambient temperature distributions for a vide values of the Prandtl number between 0.1 and 20. Further, Venkatachala et al. [3] solved the complete set of governing partial differential equations for the problem of an isothermal wall in linearly stratified atmosphere using the finite difference method. They also used series expansion and local nonsimilarity methods. Kulkarni et al. [4] investigated the problem of natural convection from an iso-thermal flat plate suspended in a stable linearly stratified fluid medium using the VonKaraman-Pohlhausen integral method. Unsteady natural convection flow over a vertical flat plate embedded in a stratified medium has been studied by Tripathi and Nath [5]. Transient numerical study of double-diffusive free convection from a vertical surface in a thermally stratified medium is highlighted by Srinivasan and Angirasa [6], and Angirasa and Srinivasan [7] and boundary layer equations were solved using an explicit finite-difference method. Later, Srinivasan and Angirasa [8] studied the unsteady laminar axisymmetric plumes that emanate from a source of combined buoyancy due to simultaneous heat and mass diffusion in thermally stratified medium and solved the boundary layer equations using an explicit finite-difference method. Also, authors [6-8] discussed the problem of two buoyancy-driving forces which are aid and oppose each other. Takhar et al. [9] presented a study on the steady natural convection boundary layer flow over a continuously moving isothermal vertical surface immersed in thermally stratified medium. The non-linear coupled partial differential equations governing the non-similar flow have been solved numerically using an implicit finite difference scheme. For small values of the streamwise distance, the governing equations have been solved by using a perturbation expansion technique along with the Shanks transformation. Later, Chamkha [10] has numerically studied the problem of steady laminar natural convection flow along a vertical permeable surface immersed in a thermally stratified environment in the presence of magnetic field and heat absorption effects. Shapiro et al. [11] studied the transient natural convection of driven flow along an infinite vertical 
plate immersed in a thermally stratified medium and considered flows induced by a sudden (impulsive) change in the temperature of the plate and a sudden application of a heat flux at the plate. Exact solutions of the governing equations were obtained by the method of Laplace transforms for the case when the Prandtl number is unity. Recently Saha et al. [12] investigated the two dimensional steady flow with heat and mass transfer effects along an isothermal vertical flat plate in a thermally stratified fluid. Boundary layer equations were solved by using an implicit finite difference method as well as local non-similarity method.

We notice to this end that natural convection flow over a vertical surface embedded in porous media also occurs in several engineering problems such as those encountered in the design of pebble-bed nuclear reactors, catalytic reactors, and compact heat exchangers, in geothermal energy conversion, in the use of fibrous materials, in the thermal insulation of buildings, in the heat transfer from storage of agricultural products which generate heat as a result of metabolism, in petroleum reservoirs, in nuclear wastes, etc. Several papers were also published on this topic but we will not present these papers here.

Since 1953, several authors [13]-[22] have developed similarity/non-similarity solutions for axi-symmetrical problems for natural convection flows over a vertical cone in steady state. Recently, Bapuji et al. [23] have numerically studied the solutions of steady flows past plane/axisymmetrical shape bodies. Also, Bapuji et al. [24, 25] have numerically studied the problem of transient natural convection from a vertical cone with non-isothermal surface temperature or non-uniform surface heat flux using an implicit finitedifference method. However, very few investigations were developed for natural convection from a vertical cone in a thermally stratified medium. Tripathi et al. [26] studied the steady non-similar free convection boundary layer flow over a vertical cone embedded in a stratified medium. Numerical results were presented for two values of the Prandtl number of 0.72 (air) and 6.0 (water) and for different values of the semi vertical angle and stratification parameter. Recently, Hossain et al. [27] studied non-similarity solutions for the problem of steady, laminar, natural convective flow and heat transfer from a vertical circular cone immersed in a thermally stratified medium with either a uniform surface temperature or a uniform surface heat flux. A finite-difference method, a series solution method and an asymptotic solution have been obtained for the non-similarity boundary layer equations. It is worth mentioning that free convection flows, which arise in a thermally stratified medium are of practical importance and in many engineering applications, cone shaped bodies are often used.

By our best knowledge, studies on unsteady laminar natural convection from a vertical cone in thermally stratified medium have not received any attention in literature. Hence, the present work is devoted to the transient laminar free convection from a vertical cone in a thermally stratified medium. The governing boundary layer equations are solved using an implicit finitedifference scheme of Crank-Nicolson type for various values of the Prandtl number Pr and stratification parameter. In order to check the accuracy of the numerical results, the present results are compared with the available results of Hering [15] and Hossian et al. [27]. Results are found to be in excellent agreement.

\section{MATHEMATICAL ANALYSIS}

Consider the problem of unsteady laminar natural convection flow of a viscous and incompressible fluid along a vertical cone placed in a thermally stratified medium. It is assumed that the viscous dissipation effects and the pressure gradient along the boundary layer are neglected. It is also assumed that initially ( $\left.t^{\prime} \leq 0\right)$, the cone surface and the surrounding fluid are in rest and have the same temperature $T_{\infty, x}^{\prime}$. Then at time $t^{\prime}>0$, the temperature of the surface of the cone is suddenly raised from $T_{\infty, x}^{\prime}$ to $T_{w}{ }^{\prime}$ and is maintained at this temperature. The co-ordinate system is chosen (as shown in Fig.1) such that $x$ measures the distance along the surface of the cone from the apex $(x=0)$ and $y$ measures the distance normal to it in the outward direction, as shown in Fig. 1. Here $\phi$ is the semi-vertical angle of the cone and $r$ is the local radius of the cone.

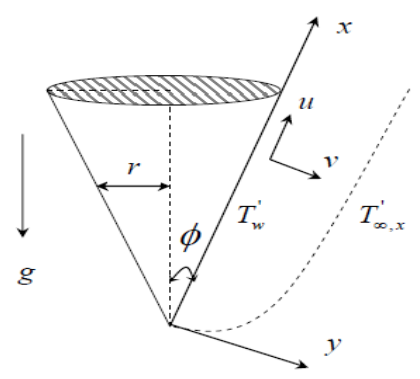

Fig. 1. Physical model and co-ordinate system

The fluid properties are assumed constant except for density variations, which give rise to the buoyancy forces in the momentum equation. The governing boundary layer equations of continuity, momentum and energy under Boussinesq approximation are as follows:

Equation of continuity:

$$
\frac{\partial u r}{\partial x}+\frac{\partial v r}{\partial y}=0
$$

Equation of momentum:

$$
\frac{\partial u}{\partial t^{\prime}}+u \frac{\partial u}{\partial x}+v \frac{\partial u}{\partial y}=g \beta \cos \phi T^{\prime}-T_{\infty, x}^{\prime}+v \frac{\partial^{2} u}{\partial y^{2}}
$$

Equation of energy:

$$
\frac{\partial T^{\prime}}{\partial t^{\prime}}+u \frac{\partial T^{\prime}}{\partial x}+v \frac{\partial T^{\prime}}{\partial y}=\alpha \frac{\partial^{2} T^{\prime}}{\partial y^{2}} .
$$

Subject to the initial and boundary conditions are 


$$
\begin{aligned}
& t^{\prime} \leq 0: \quad u=0, \quad v=0, \quad T^{\prime}=T^{\prime}, \quad \text { for } \text { all } x, y \\
& t^{\prime}>0: \quad u=0, \quad v=0, \quad T^{\prime}=T^{\prime}{ }_{w} \quad \text { at } \quad y=0 \\
& u=0, \quad T^{\prime}=T^{\prime}, \quad \text { at } \quad x=0 \\
& u \rightarrow 0, \quad T^{\prime} \rightarrow T^{\prime}, \quad \text { as } \quad y \rightarrow \infty
\end{aligned}
$$

where $T_{\infty, 0}^{\prime}$ is the constant temperature at the vertex of the cone.Further, we introduce the following non-dimensional quantities:

$$
\begin{gathered}
X=\frac{x}{L}, \quad Y=\frac{y}{L} G r_{L} \frac{1}{4}, \quad R=\frac{r}{L}, \quad \mathrm{U}=\frac{u L}{v} G r_{L} \frac{-1}{2}, \\
V=\frac{v L}{v} G r_{L} \frac{-1}{4}, \quad t=\frac{v t^{\prime}}{L^{2}} G r_{L} \frac{1}{2}, T=\frac{T^{\prime}-T_{\infty, x}^{\prime}}{T_{w}^{\prime}-T_{\infty, 0}^{\prime}} .
\end{gathered}
$$

Where $G r_{L}=g \beta \cos \phi T_{w}^{\prime}-T_{\infty, 0}^{\prime} L^{3} / v^{2}$ is the Grashof number defined based on $L, \operatorname{Pr}=v / \alpha$ is the Prandtl number, $r=x \sin \phi, \quad S \quad=1 / \Delta t_{0} \quad d T_{\infty, x}^{\prime} / d X \quad$ is the thermal stratification parameter and $\Delta t_{0}=T^{\prime}{ }_{w}-T_{\infty, 0}^{\prime}$.

We assume that the temperature of the stratified medium $T^{\prime}{ }_{\infty, x}$ is a linear function of the coordinate $X$ of the form $\quad T_{\infty, x}^{\prime}=T_{\infty, 0}^{\prime}+S X \quad$ where $S=\left(d T_{\infty, x}^{\prime} / d X\right) / \Delta t_{0}>0$ for a stable stratified medium. We notice that for the linear thermal stratification $S$ is a constant and for other variation it can be represented as a function of $X$. Using (5), Eqs. (1) - (3) can be reduced to the following non-dimensional form:

$$
\begin{gathered}
\frac{\partial U R}{\partial X}+\frac{\partial V R}{\partial Y}=0 \\
\frac{\partial U}{\partial t}+U \frac{\partial U}{\partial X}+V \frac{\partial U}{\partial Y}=T+\frac{\partial^{2} U}{\partial Y^{2}} \\
\frac{\partial T}{\partial t}+U \frac{\partial T}{\partial X}+V \frac{\partial T}{\partial Y}+S U=\frac{1}{\operatorname{Pr}} \frac{\partial^{2} T}{\partial Y^{2}}
\end{gathered}
$$

Subject to the corresponding initial and boundary conditions

$$
\begin{aligned}
t \leq 0: U & =0, V=0, T=0, \text { for all } X \text { and } Y, \\
t>0: U & =0, V=0, T=1-S X, \text { at } Y=0, \\
U & =0, T=0, \text { at } X=0, \\
U & \rightarrow 0, T \rightarrow 0, \text { as } Y \rightarrow \infty .
\end{aligned}
$$

\section{NUMERICAL PROCEDURE}

The unsteady, non-linear and coupled partial differential equations (6) - (8) with the initial and boundary conditions (9) are solved numerically employing a finite-difference scheme of Crank-Nicolson type as described in detail by Bapuji et al. [24]. The finite- difference scheme for Eqs. (6) - (8) is reduced to tri-diagonal system of equations and is solved by Thomas algorithm as discussed in Carnahan et al. [28]. The local truncation error is $O \Delta t^{2}+\Delta Y^{2}+\Delta X$ and it tends to zero as
$\Delta t, \Delta Y$ and $\Delta X$ tend to zero. Therefore, the scheme is compatible. The finite-difference scheme is unconditionally stable as explained by Bapuji et al. [24]. Stability and compatibility ensure the convergence.

\section{RESULTS AND DISCUSSION}

In order to prove the accuracy of our numerical results, the present results for the steady- state flow at $X=1.0$ are compared with available solutions from the open literature.

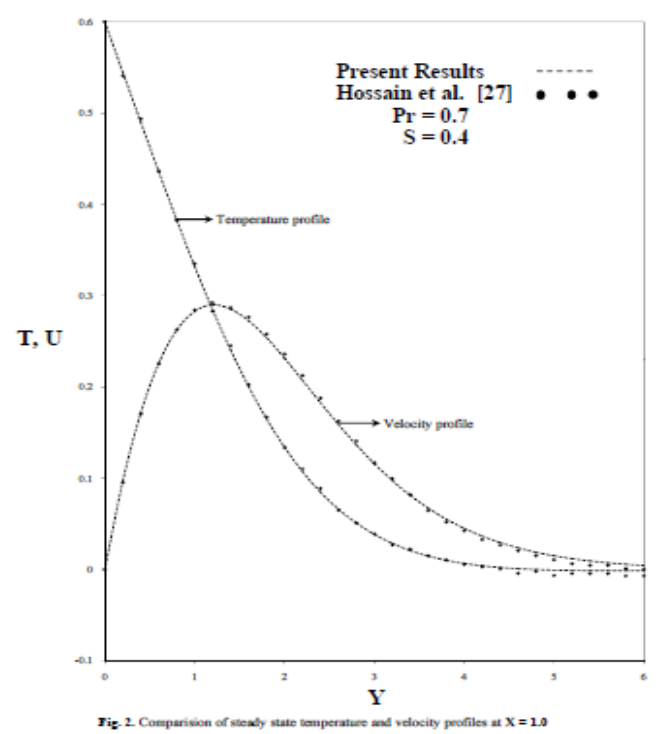

Thus, the velocity and temperature profiles for a cone with uniform surface temperature when $\operatorname{Pr}=0.7$ and stratification parameter $S=0.4$ are compared with the non-similarity solutions of Hossain et al. [27] when the Prandtl number of $\operatorname{Pr}=0.7$ and the stratification parameter of $S=0.4$. These profiles are displayed in Fig. 2. It is seen that the agreement between these results is excellent. In the present investigation, the maximum value of $X$ at $X=1.0$ has been considered because for higher values of $X$ the laminar flow may not be valid. However, it is clear that for $S=1$, the ambient temperature is equal to the wall temperature at $X=1$.

For $S=0$ (un-stratified medium), the numerical results are the same with those obtained by Bapuji et al. [24] for an isothermal vertical cone. Also, Bapuji et al. [24] proved that their results agree very well with those of Hering [15] for the surface temperature of both isothermal and non-isothermal vertical cone in steady-state flows.

It should be noticed that the practical limit for the upward boundary layer flow occurs only when the stratified fluid is stable, i.e., $S<1$. When $S>1$, the buoyancy forces act downward as the ambient fluid is hotter than the heated cone surface. Because of this fact, the present results are valid only for $S<1$.

Figures 3-6 show the transient velocity and temperature profiles at $X=1.0$ for some values of the parameters $\operatorname{Pr}$ 
and $S$. The value of $t$ with the symbol $(*)$ denotes the time taken to reach the steady-state flow. In Figs.3 and 4 , the transient velocity and temperature profiles are plotted for various values of $\operatorname{Pr}$ when $S=0.4$.
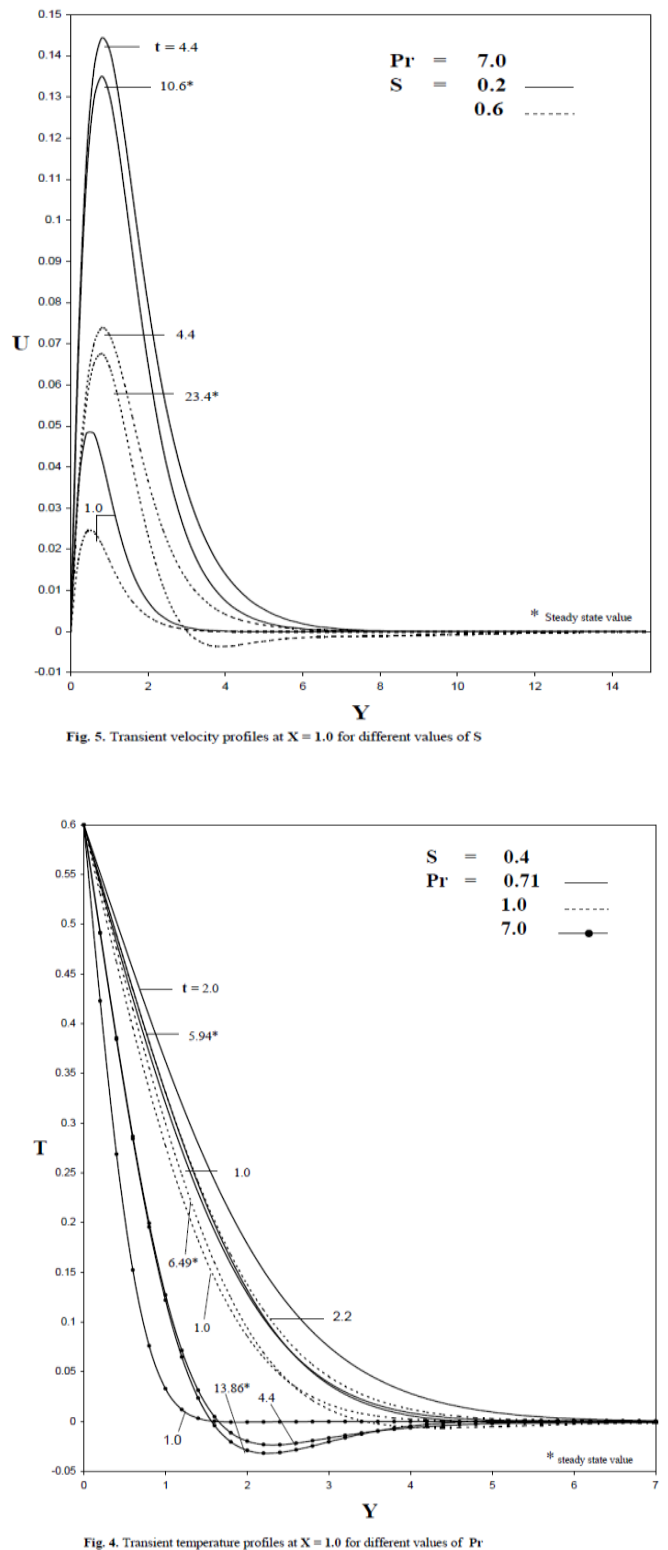

It is seen from these figures, as expected, that the increase of Pr causes a reduction in the velocity and temperature profiles. It is also observed from these figures that the difference between temporal maximum values and steadystate values are reduced when $\mathrm{Pr}$ increases. Further, it is noticed that the time taken to reach the steady-state flow increases, the thermal boundary layer and momentum boundary layer thickness reduces with increasing Pr.

It is also observed from Fig. 4 that negative values of the non-dimensional temperature appear as wings of the profiles in the outer region with increasing $\operatorname{Pr}$.

The transient velocity and temperature profiles for various values of $S$ with $\operatorname{Pr}=7.0$ are shown in Figs. 5 and 6. It can bee seen that an increase in the ambient thermal stratification parameter invariably decreases the thermal buoyancy. Hence, the decrease in the velocity levels with increase in thermal stratification is fairly obvious. For $S<1$, there is a positive buoyancy at $X=1$, but negative values of the non-dimensional temperature profiles are still observed in the outer region. For high values of $S$, the temperature in the ambient medium increases rapidly with the height and the fluid coming up will be much cooler in the outer region of the boundary layer. Hence, negative values of non-dimensional temperature appear in the "wings" of profile. This occurrence is often referred to as "temperature defect" in the flow and it is also described by many investigators in the literature [2, 6, 7, 8 and 12].
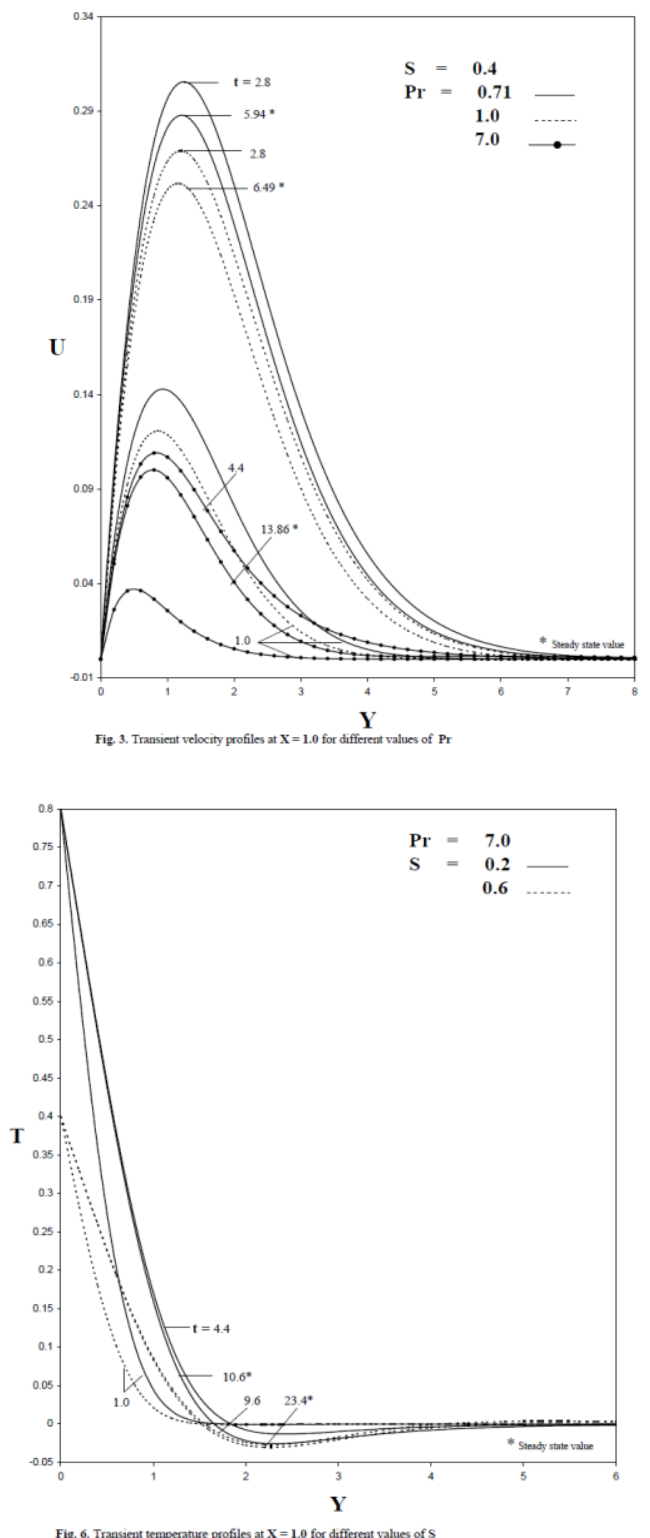

Also, observed from Figs. 5 and 6 is the difference between temporal maximum values and the steady-state values, which decrease with increasing the stratification parameter $S$. It is also observed that as $S$ increases, the velocity and temperature reduce throughout the transient period and the time taken to reach steady state value increases 


\section{CONCLUSIONS}

This paper deals with the unsteady laminar free convection flow along a vertical cone in a stable thermally stratified medium. The dimensionless governing boundary layer equations are solved numerically using an implicit finitedifference method of Crank-Nicolson type. Present results are compared with available results from the open literature and are found to be in very good agreement. The following conclusions are drawn:

- The velocity and temperature profiles reduce when the parameters $S$ and Pr are increased.

- The momentum boundary layers become thinner when the values of $S$ and Prare increased.

- The thermal boundary layer becomes thicker when Pr is reduced.

- The time taken to reach the steady-state values are increases with increasing $\operatorname{Pr}$ and $S$.

- Due to "temperature defect" in the flow, the profiles of velocity and temperature appear as wings in the outer region.

- The difference between the values of temporal maximum velocity/temperature and the values of the steady-state become more when $\operatorname{Pr}$ and $S$ are decreased.

\section{REFERENCES}

[1] R. Cheesewright," Natural Convection from a Plane Vertical Surface in Non-Isothermal Surroundings", Int. J. Heat Mass Transfer, vol. 10 , Pp. $1847-1859,1967$

[2] A K. T. Yang, J. L. Novotny and Y. S. Cheng," Laminar Free Convection from a Nonisothermal Plate Immersed in a Temperature Stratified Medium", Int. J. Heat Mass Transfer, vol. 15, Pp. 1097-1109, 1972 .

[3] B. J. Venkatachala and G. Nath," Nonsimilar Laminar Natural Convection in a Thermally Stratified Fluid", Int. J. Heat Mass Transfer,vol. 24, Pp. 1848- 1850, 1981

[4] A. K. Kulkarni, H. R. Jacobs and J. J. Hwang," Similarity Solution For Natural Convection Flow Over an Isothermal Vertical Wall Immersed in Thermally Stratified Medium", Int. J. Heat Mass Transfer, Vol. 30, Pp. 691-698, 1987

[5] R.K. Tripathi and G. Nath, "Unsteady Natural Convection Flow Over a Vertical Plate Embedded in a Stratified Medium", Int. J. Heat Mass Transfer Vol. 36, Pp. 1125-1128,1993.

[6] J. Srinivasan and D. Angirasa," Numerical Study Of Double-Diffusive Free Convection from a Vertical Surface", Int. J. Heat Mass Transfer,Vol. 31, Pp. 2033-2038,1998.

[7] D. Angirasa and J. Srinivasan," Natural Convection Flows Due to the Combined Buoyancy Of Heat and Mass Diffusion in Thermally Stratified Medium", ASME Journal of Heat Transfer, Vol. 111, Pp. 657$663,1989$.

[8] J. Srinivasan and D. Angirasa," Laminar Axisymmetric Multicomponent Buoyant Plumes in a Thermally Stratified Medium”, Int. J. Heat Mass Transfer ,Vol. 33, Pp. 1751-1757.,1990

[9] H. S. Takhar, A. J. Chamkha and G. Nath," Natural Convection Flow from a Continuously Moving Vertical Surface Immersed in a Thermally Stratified Medium” "Heat and Mass Transfer, ,Vol. 38 ,Pp. 17-24 ,2001.

[10] Ali. J. Chamkha, "Laminar Hydromagnetic Natural Convection Flow Along a Heated Vertical Surface in a Stratified Environment with Internal Heat Absorption, Can. J. Phys. Vol. 80 ,Pp. 1145-1155, 2002.

[11] A. Shapiro and E. Fedorovich," Unsteady Convectively Driven Flow Along a Vertical Plate Immersed in a Stably Stratified Fluid", J. Fluid Mech. Vol. 498 ,Pp. 333-352,2004.

[12] S. C. Saha and M. A. Hossain," Natural Convection Flow with Combined Buoyancy Effects Due to Thermal and Mass Diffusion in a
Thermally Stratified Media, Nonlinear Analysis": Modelling and Control,Vol. 9(1), Pp. 89-102, 2004.

[13] H. J. Merk and J.A. Prins," Thermal Convection Laminar Boundary Layer-I, II", Appl. Sci. Res. A4 (1953) 11-24 and (1954) 195-206.

[14] R. G. Hering and R. J. Grosh," Laminar Free Convection from a NonIsothermal Cone”, Int. J. Heat Mass Transfer ,Vol. 5 ,Pp. 1059-1068 ,1962.

[15] R. G. Hering," Laminar Free Convection from a Non-Isothermal Cone at Low Prandtl Number", Int. J. Heat Mass Transfer, Vol. 8, Pp. 13331337,1965 .

[16] M. Alamgir,"Overall Heat Transfer from Vertical Cones in Laminar Free Convection: an Approximate Method", ASME Journal of Heat Transfer, Vol. 101, Pp. 174-176,1989.

[17] R. S. R. Gorla and Robert A. Startman," Natural Convection Boundary Layer Flow of Water at $4^{0} \mathrm{C}$ Past Slender Cones", Int. Comm. Heat Mass Transfer", ,vol 13, Pp403-411,1986

[18] I. Pop and H.S. Takhar," Compressibility Effects in Laminar Free Convection from a Vertical Cone”,Appl.Sci.Res.Vol. 48,Pp. 71-82,1991.

[19] M. A. Hossain and S. C. Paul, "Free Convection from a Vertical Permeable Circular Cone with Non-Uniform Surface Heat Flux", Heat Mass Transfer,Vol. 37, Pp. 167-173,2001.

[20] M. A. Hossain and S. C. Paul, "Free Convection from a Vertical Permeable Circular Cone With Non-Uniform Surface Heat Flux", Heat Mass Transfer,Vol. 37, Pp. 167-173,2001.

[21] I. Pop, T. Grosan and M. Kumari," Mixed Convection Along a Vertical Cone for Fluids of Any Prandtl Number Case of Constant Wall Temperature", Int. J. Of Numerical Methods For Heat and Fluid Flow, Vol. 13 Pp. 815-829, 2003.

[22] H. S. Takhar, A. J. Chamkha and G. Nath," Effect of Thermo-Physical Quantities on the Natural Convection Flow of Gases Over a Vertical Cone", Int. J. Engng. Sci., Vol. 42 , Pp. 243-256, 2004.

[23] Bapuji Pullepu and K. Ekambavanan," Natural Convection Effects on Two Dimensional Axi-Symmetrical Shape Bodies (Flow past a vertical/cone/thin cylinder) and Plane Shape Bodies (Flow over a vertical/inclined/ horizontal plates)". In: Proceedings of $33^{\text {rd }}$ National and $3^{\text {rd }}$ International Conference on Fluid Mechanics and Fluid Power, December 7-9, 2006, IIT, Bombay, India.(Paper No 1707).

[24] Bapuji Pullepu, K. Ekambavanan and I. Pop," Finite Difference Analysis of Laminar Free Convection Flow Past a Non Isothermal Vertical Cone", Heat Mass Transfer (article in press DOI: 10.1007/s00231-0070266-3).

[25] Bapuji Pullepu, K. Ekambavanan and I. Pop,” Transient Laminar Free Convection from a Vertical Cone with Non-Uniform Surface Heat Flux", Studia Univ. Babes-Bolyai Mathematica (in press, 2007).

[26] R. K. Tripathi, A. Sau and G. Nath," Laminar Free Convection Flow Over a Cone Embedded in a Stratified Medium", Mech. Res. Commun. ,Vol. 21, Pp. 289-296,1994.

[27] M. A. Hossain, S. C. Paul and A. C. Mandal," Natural Convection Flow Along a Vertical Circular Cone with Uniform Surface Temperature And Surface Heat Flux In A Thermally Stratified Medium”, Int. J. Numerical Methods for Heat \& Fluid Flow, Vol. 12, Pp. 290-305, 2002.

[28] B. Carnahan, H. A. Luther and J. O. Wilkes," Applied Numerical Methods", John Wiley and Sons, New York, 1969. 